\title{
La visión de conservación del recurso forestal: una perspectiva basada en la legislación forestal y ambiental de Nicaragua
}

\section{The vision of conservation of the forest resource: a perspective based on the forestry and environmental legislation of Nicaragua}

\author{
Álvaro Noguera-Talavera ${ }^{1}$, Gladys Cruz $^{2}$ \\ ${ }^{1}$ Docente UNA. Punto Focal Red Latinoamericana de Derecho Forestal y Ambiental. \\ ${ }^{2}$ Procuradora Ambiental. Licenciada en Derecho. Asesora Legal del Ministerio Agropecuario y Forestal.
}

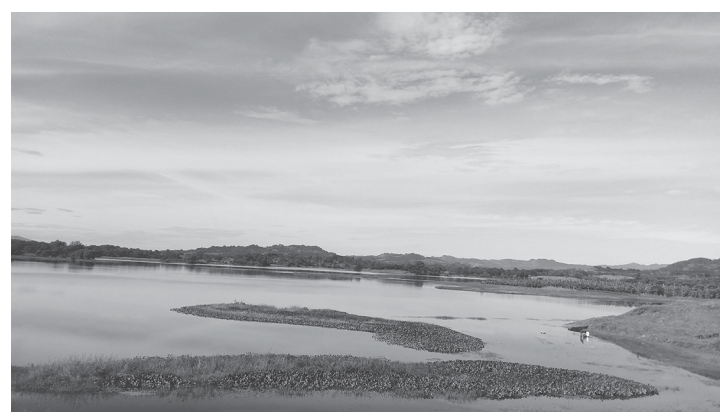

\section{RESUMEN}

Este artículo es una síntesis basada en la legislación ambiental y forestal nicaragüense. El objetivo fue presentar una perspectiva de la visión y eficiencia de ambos instrumentos jurídicos para la conservación de los ecosistemas forestales. La metodología para la preparación de este trabajo consistió en la revisión critica de tres leyes (Ley 647 de Reformas y Adiciones a la ley 217; Ley General del Medio Ambiente y los Recursos Naturales, la ley 462: Ley de Conservación, Fomento y Desarrollo Sostenible del Sector Forestal; y ley 585 Ley de Veda para el Corte, Aprovechamiento y Comercialización del Recurso Forestal), así como los reglamentos y normas técnicas de dichas leyes considerando los elementos jurídicos que de manera general (a nivel de ecosistema) como particular (a nivel de especies) establecen medidas de conservación. Desde el punto de vista de conservación per se, es la ley 647 la que contiene mayores elementos jurídicos relativos a la conservación del recurso forestal, estableciendo, por un lado, instancias facultadas para aprobar, supervisar y evaluar actividades con potencial degradante y propuestas de conservación de los ecosistemas forestales, normas generales para la protección de suelos forestales y ciertos elementos para la creación de mecanismos de acceso a incentivos de tipo fiscal. Un punto importante derivado de la ley 462 es el reglamento de áreas protegidas cuya visión gira alrededor de la restricción de uso de ecosistemas, y no así de conservar los ecosistemas forestales o especies de importancia ecológica, productiva o representativos. $\mathrm{Al}$ analizar elementos contenidos en la ley 462 se percibe que tanto esta como su reglamento y normas técnicas hacen mayor énfasis en la definición de ecosistemas para producción, y establecimiento de las reglas de carácter técnico para el manejo; lo más relevante en cuanto a conservación, la restricción de aprovechamiento en áreas de protección municipal, y zonas fronterizas; lo cual es ratificado en la ley 585 o de veda cuya acción gira en torno a la protección de seis especies con niveles poco documentadas de amenaza, y excepciones en ciertos territorios, lo que al final no contribuye a la conservación

\section{ABSTRACT}

This paper is an abstract based on environmental and forest Nicaraguan legislation. The objective was deliver a perspective of the approach and efficiency of these laws to improve the forest ecosystems conservation. The methodology to prepare it consisted in the critical review of three laws (Law 647: General law of environment and natural resources, law 462: Law of conservation, promotion and sustainable development of forest activities; and law 585: Law of restriction to cut, logging and commercialization of forest resource) thus complementary legal instruments of these laws takin account the legal elements that consider a general approach (ecosystem level) as well as particular (species level) useful to forest conservation strategies. Based on per se conservation point of view, the law 647 has many legal elements relate with forest resource conservation, emphasizing in institutional role to confer, to observe and to assess forest activities with negative potential impact, and conservation proposals of ecosystems forest, normative to forest soil protection and some elements to create the orientation to taxes incentives. The regulation of protected areas, is an important point of the law 462 being the approach to restrict the use and management of the ecosystems, contrary to needed of conserve ecosystems or species with ecological importance or representative. The analysis of the law 462 suggest that the normative emphasize in the definition of ecosystem to wood production and forest management; the restriction of logging in municipal protected areas and border zone, which is found in law 585 (also known as close law) that pretend to protect six forest species with undocumented threat, and unrestricting y some areas. The conservation perspective of Nicaraguan legislation content restrictive elements, while some regulations pretend sustainable use, but without orientation to application and assessment.

Keywords: Nicaraguan legislation, ecosystems use restriction, forest ecosystems. 
de dichas especies. La visión de conservación contenida en la legislación Nicaragüense expresa mayormente aspectos restrictivos, mientras otro grupo de normas persigue el uso sostenible, pero sin reglamentaciones claras e instrumentos para gestión y evaluación.

Palabras clave: legislación nicaragüense, restricción de uso de ecosistemas, ecosistemas forestales

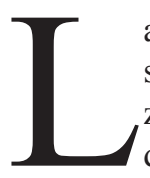

a conservación es una disciplina dedicada a la preservación, rescate, mantenimiento, estudio y utilización del patrimonio que representa la biodiversidad (Pezoa 2001).

La conservación puede planificarse y realizarse bajo diferentes enfoques y estrategias que hacen uso de una variedad de actividades en particular. El alcance o dimensión de los enfoques corresponden a visiones tradicionales y tendencias actuales determinadas por el nivel de amenaza. Los enfoques más comunes en relación a la conservación de la biodiversidad son: el enfoque por especies y el enfoque por ecosistemas. Ambos enfoques utilizan estrategias como la conservación in situ y conservación ex situ para lograr los objetivos de conservación definidos por el Convenio sobre Diversidad Biológica. Estas dos estrategias son complementarias y permiten garantizar la conservación del patrimonio genético de las especies y sus poblaciones, en el mediano y largo plazo.

De acuerdo con Pezoa (2001), la conservación debe planificarse de tal modo que se integre con los planes de desarrollo sustentable y de utilización sostenible de los recursos naturales de las diversas regiones de un país. Esta integración sería la única garantía que permita mantener los objetivos de conservar la biodiversidad en el tiempo.

La legislación ambiental y forestal nicaragüense tiene como misión aportar al desarrollo del país a través del establecimiento de normas legales para el buen aprovechamiento de los recursos naturales, principalmente en lo relativo a los bienes como la madera y otros productos del bosque, para alcanzar así el precepto de conservación.

El presente trabajo tiene como propósito presentar una perspectiva de la incorporación y eficiencia de elementos jurídicos a las iniciativas de conservación del recurso forestal bajo el enfoque de manejo-aprovechamiento de los recursos forestales.

Objeto e implicaciones del término conservación. Un primer elemento objeto de conservación son los recursos naturales, término que en la ley ambiental nicaragüense es definido como "elementos naturales de que dispone el hombre para satisfacer sus necesidades económicas, sociales y culturales". Así mismo se definen como "elementos naturales susceptibles de ser aprovechados por el hombre".

También se incluye como parte de las adiciones a dicha ley el termino recursos naturales renovables, que es definido como: "aquellos que tienen la capacidad de regenerarse por procesos naturales y que pueden también, ser mantenidos o incrementados por el manejo que el ser humano haga de ellos".

A este tipo de recursos pertenece el agua, el suelo, el aire, la energía en todas sus formas, la biomasa constituida por la flora y la fauna, tanto silvestre como doméstica.

En un segundo nivel derivado de los recursos naturales y que son de interés se presentan los recursos forestales. En cuanto a la definición de "recurso forestal", este no es definido en ninguno de los instrumentos jurídicos de la legislación forestal, abordándose en su conjunto a partir de los siguientes términos que se presentan en el reglamento de la ley 462 (decreto ejecutivo $\mathrm{N}^{\circ}$ 73-2003):

Vuelo forestal. Todos los árboles, arbustos, plantas leñosas y demás especies vegetales a partir de la superficie del suelo.

Área boscosa. Extensión de tierra que cuenta con cobertura forestal maderable, al menos en un $30 \%$.

Vegetación forestal. Conjunto de plantas dominadas por especies arbóreas, arbustivas o crasas, que crecen y se desarrollan en forma natural formando bosques, selvas y vegetación de zonas áridas.

Terrenos forestales. Toda área cubierta con bosque, comprende primario, secundario, los matorrales y tacotal y que tenga vocación forestal, excluyendo las áreas urbanas.

En tal sentido, la ley hace mención como figura legal de régimen de tenencia del recurso forestal y responsabilidades al dueño del vuelo forestal (como sinónimo de recurso forestal), siendo este el objeto para la aplicación de medidas de conservación a partir del manejo forestal.

Bajo el contexto de conservación promovido por el Convenio sobre Biodiversidad, la indefinición de lo que realmente implica el recurso forestal, sugiere una falta de visión eco sistémica (complejidad, dinámica y evolución) de manejo y sostenibilidad; mientras las definiciones solo enfatizan en aspectos como cobertura forestal maderable y vocación forestal; por lo que las estrategias de conservación apuntan a una conservación basada en actividades extractivas, cuyo precepto para conservar es la planificación para generar bajo impacto; que tiene como principal debilidad la carencia de un eficiente sistema de monitoreo en la fase de post aprovechamiento para evaluar los daños e implementar planes de restauración o conservación de especies o del ecosistema aprovechado . 
El termino conservación, desde el punto de vista de la definición que se presenta en la ley 217 , ley 647 y el reglamento de la ley 462 (decreto 73-2003) consiste en "la aplicación de las medidas necesarias para preservar, mejorar, mantener, rehabilitar y restaurar las poblaciones, y los ecosistemas, sin afectar su aprovechamiento".

En lo general, el término es inclusivo de una serie de procesos de carácter técnico y legal, y abarca una gama de opciones para considerar como meta los procesos que integran el enfoque de conservación; sin embargo, la falta de énfasis en la elaboración de mecanismos e instrumentos claros para implementar las actividades contenidas en la definición crea un vacío; que a largo plazo no permite cumplir, ni monitorear y evaluar medidas encaminadas a la conservación del recurso bosque.

Objetos de los instrumentos jurídicos y su visión para la conservación del recurso forestal. La visión de conservación expresada en los instrumentos jurídicos analizados es plasmada en los objetos, que literalmente expresan:

Ley 217. Establecer las normas para la conservación, protección, mejoramiento y restauración del medio ambiente y los recursos naturales que lo integran, asegurando su uso racional y sostenible, de acuerdo a lo señalado en la Constitución Política.

Ley 462. Establecer el régimen legal para la conservación, fomento y desarrollo sostenible del sector forestal, tomando como base fundamental el manejo forestal del bosque natural, el fomento de las plantaciones, la protección, conservación y la restauración de áreas forestales.

Ley 585. Establecer una veda por un período de 10 años, para el corte, aprovechamiento y comercialización de árboles de las especies de caoba, cedro, pochote, pino, mangle y ceibo en todo el territorio nacional, que podrá ser renovable por períodos similares, menores o mayores.

En los dos primeros instrumentos es de particular interés resaltar que las visiones apuntan hacia principios y tendencias actuales de conservación en el sentido que el uso racional debe conducir hacia la protección, mejoramiento y aplicación de medidas de recuperación, logrando así la sostenibilidad del recurso en el tiempo.

En lo específico, el objeto de la Ley 462 establece el manejo forestal como el enfoque o ruta a través de la cual es posible alcanzar el desarrollo del sector forestal; que en la realidad no corresponde solamente al bosque, sino a cada uno de los actores de la cadena productiva, transformación, comercialización y reinversión o distribución equitativa de los bienes que genera el bosque; por lo que mientras algunos de los actores de cada eslabón no estén empoderados de su papel, esta visión se queda en lo abstracto.

Por otro lado, en lo que se refiere al alcance de las metas del manejo forestal frente a la conservación del bosque, pocas experiencias en Nicaragua muestran bajo impacto y continuidad en medidas como reposición del bosque, manejo silvicultural post aprovechamiento, restauración, planes para conservación de especies emblemáticas o de interés comercial, entre otras.

En síntesis el manejo forestal -desde la práctica común Nicaragüense- es un proceso de carácter técnico cuyo principio denota elementos de sostenibilidad, pero desde la perspectiva de su implementación es solo una herramienta constituida por un conjunto de formatos de actividades técnicas (inventario forestal, mapeo, proyecciones de rendimiento y cumplimiento de normas técnicas obligatorias sin un verdadero análisis de sostenibilidad) para lograr la obtención de una concesión de aprovechamiento anual que justifique la inversión de capital que solo extrae y no trabaja por recuperar el bosque.

En cuanto a la relación beneficios y sostenibilidad del recurso forestal, es necesario resaltar que el mayor potencial se encuentra en la costa Caribe de Nicaragua; sin embargo, las comunidades indígenas son las que menos aprovechan estos recursos naturales. En la práctica se observa que a pesar que los recursos son aprovechados, no existe desarrollo económico en las comunidades, por cuanto son dadas en concesión a inversionista fuera de las comunidades que solo explotan el recurso sin realizar inversión en la zona.

De manera contraria, la ley 585 establece restricción en el aprovechamiento como visión alternativa para lograr la conservación de especies de importancia económica y ecológica para un periodo en particular; anteponiéndose así a las visiones reales de conservación que establecen la necesidad de crear sistemas de muestreo a largo plazo de las poblaciones, definición de áreas de conservación, desarrollo de planes de conservación de las especies con alto grado de amenaza y fácil acceso a los comunitarios.

De lo antes descrito es posible identificar aspectos comunes en las visiones de los instrumentos jurídicos relativos a la conservación; sin embargo, es evidente que los enfoques básicos en los cuales se basan dichas visiones son asumidas por las entidades gubernamentales como practicas puntuales y no como enfoques holísticos e integradores que aseguren la conservación del recurso forestal.

Aspectos de conservación-protección. Desde el punto de vista de operativización de lo establecido en materia de protección para evitar la degradación y extinción de recursos naturales; así como mejoramiento y recuperación del medio ambiente; el artículo 12 de la Ley 217 y sus reformas y adiciones establece responsabilidades de su planificación a todos los organismos de la administración pública desde las competencias que establece la Ley 290; sin embargo, es el Ministerio del Ambiente y los Recursos Naturales el ente con mayor competencia en los aspectos de este acápite.

Desde otra perspectiva de competencias, el Instituto Nacional Forestal es un ente únicamente facultado para ejercer vigilancia o inspeccionar, aprobar permisos, evaluar planes de manejo forestal y disponer las medidas, correcciones y sanciones a violaciones de conformidad a lo establecido en 
la ley 462 y su reglamento. En tal sentido, se establece que actividades de protección y conservación serán coordinadas con otras entidades del sector público relacionadas y con la participación del sector privado.

Un elemento de relevancia en cuanto a la conservación desde una visión de ecosistema es el abordado en el artículo 108 de la ley 217 en lo referido a las normas que restringen el cambio de uso en suelos con vocación forestal; aun cuando sea para el establecimiento de plantaciones forestales, cuya visión no debe ser aceptada dentro del marco de programas de restauración de bosques, pero que debe tener apertura hacia medidas de rehabilitación de bosques alterados por aprovechamiento.

Otro elemento valedero es la definición de instrumentos como el estudio y la evaluación de impacto ambiental cuyo carácter técnico sugieren un precepto de previsión de impactos a recursos forestales $u$ otros asociados al bosque; adquiriendo así un carácter vinculante o contractual para la adquisición de compromisos de implementación de medidas de control y mitigación por alteración o degradación; ejemplo de ello es el artículo 17 de la ley 462.

Por otro lado, se asumen como lineamientos propios de conservación en áreas bajo manejo la aplicación de disposiciones expresadas en los artículos 18 y 19 de la ley 462, que establecen protección especial mediante el auxilio de autoridades policiales; así como prohibición de corte, extracción o destrucción de árboles a través de la figura de veda e inclusión de especies en listados nacionales e internacionales de convenios internacionales ratificados por el país; y el traslado de facultades a las municipalidades para la creación de alternativas como las áreas forestales de protección municipal y parques ecológicos municipales.

Un ejemplo de implementación de este mecanismo es el caso donde se restringe el manejo forestal en áreas protegidas a lo dispuesto en el reglamento $\mathrm{u}$ otro instrumento jurídico relacionado a esta estrategia de conservación, mientras en la ley 585 el aprovechamiento de especies (aun las no vedadas) se prohíbe de manera indefinida.

Artículo 18. Las plantaciones forestales y las áreas de bosque natural bajo manejo privado o estatal, tendrán protección especial en caso de invasión u otras acciones ilícitas que atenten contra las mismas. Las autoridades policiales deberán prestar el auxilio correspondiente al propietario o a cualquier autoridad civil o militar que lo solicite, para proceder conforme a la ley al desalojo o a prevenir y neutralizar las actividades que destruyan o causen daños al recurso forestal.

Artículo 19. Se prohíbe el corte, extracción o destrucción de árboles de aquellas especies protegidas y en vías de extinción que se encuentren registradas en listados nacionales y en los convenios internacionales ratificados por el país. Se exceptúan los árboles provenientes de plantaciones debidamente registradas en el Registro Nacional Forestal.
La conservación del recurso forestal mediante la declaración de áreas protegidas. En Nicaragua existen actualmente un poco más de tres millones de hectáreas de cobertura forestal (INAFOR y FAO, 2007-2009) correspondiendo esta cifra al 25\% del territorio nacional. De estas, dos millones de hectáreas se encuentran dentro del Sistema Nacional de Áreas Protegidas (Rueda, 2007), y aun cuando solamente $16.9 \%$ de los ecosistemas se encuentran dentro del Sistema Nacional de Áreas Protegidas (SINAP), estas son cifras importantes desde el punto de vista de iniciativas de conservación del recurso vegetal presente en estos ecosistemas.

Sin embargo, a pesar de los muchos esfuerzos y recursos orientados a lograr un manejo sostenible de las áreas protegidas, factores externos e internos hacen que estas áreas experimenten la misma dinámica de disminución o pérdida de la biodiversidad, en comparación a las áreas fuera del SINAP de Nicaragua (Noguera-Talavera, 2010).

Los instrumentos jurídicos relacionados a la conservación del recurso forestal en áreas protegidas son la ley 647 , y el decreto $\mathrm{N}^{\circ}$ 01-2007 o reglamento de áreas protegidas de Nicaragua, que expresan las visiones de conservación basadas principalmente en la restricción de uso-aprovechamiento de especies y ecosistemas clasificados como representativos de ecorregiones; así como limitando el acceso público en general, como en el caso de las reservas biológicas.

En este caso, de las nueve categorías de manejo que constituyen el sistema nacional de áreas protegidas de $\mathrm{Ni}$ caragua, siete dirigen sus lineamientos de conservación a la protección de hábitats, ecosistemas y especies; no definiendo en la mayoría de los casos estrategias de inclusión de las comunidades como forma de asegurar la sostenibilidad de esta iniciativa.

En este sentido, es necesario traer a colación la existencia por definición en normas ecológicas tradicionales de zonas de uso en la Reserva de Biosfera BOSAWAS, en cuyo territorio persisten comunidades de las etnias Miskitu y Mayangna; que a través de su cosmovisión sobre la relación hombre-naturaleza implementan prácticas que apuntan a la sostenibilidad de los recursos naturales; pero que por contexto socioeconómico y cultural actual, no son consideradas en las estrategias o planes de manejo de esta importante área protegida, atentando así con la persistencia de especies y ecosistemas de interés para la humanidad.

\section{CONCLUSIÓN}

Sobre la base en los documentos jurídicos incluidos en este trabajo, es posible inferir una carencia de instrumentos puntuales que garanticen principios de conservación contenidos en el convenio internacional sobre conservación de la biodiversidad y la recién aprobada ley 807 (conservación y utilización de la biodiversidad) que hace énfasis es aspectos procedimentales para la obtención de beneficios socioeconómicos. 


\section{ARTILCULO DEREVISTIONN}

\section{REFERENCIAS BIBLIOGRÁFICAS}

MAGFOR (Ministerio Agropecuario y Forestal, NI). 2008. Ley No. 647, Ley de Reformas y Adiciones a la ley No. 217. Ley General del Medio Ambiente y los Recursos Naturales. La Gaceta No. 62, Diario Oficial de la Asamblea Nacional. Managua, Nicaragua. 2006. Ley No. 585, Ley de Veda para el Corte, Aprovechamiento y Comercialización del Recurso Forestal. La Gaceta No.

120, Diario Oficial de la Asamblea Nacional. Managua, Nicaragua. 2003. Ley No. 462, Ley de Conservación, Fomento y Desarrollo Sostenible del Sector Forestal. La Gaceta No. 168, Diario Oficial de la Asamblea Nacional. Managua, Nicaragua. 2003. Decreto Ejecutivo No. 73-2003. Reglamento de la Ley No. 462, Ley de Conservación, Fomento y Desarrollo Sostenible del Sector Forestal. La Gaceta No. 208, Diario Oficial de la Asamblea Nacional. Managua, Nicaragua. 1996. Ley No. 217, Ley General del Medio Ambiente y los Recursos Naturales. La Gaceta No. 105, Diario Oficial de la Asamblea Nacional. Managua, Nicaragua.

MARENA (Ministerio del Ambiente y los Recursos Naturales, NI). 2007. Decreto Ejecutivo No. 01-2007. Reglamento de Áreas Protegidas de Nicaragua. La Gaceta No. 08, Diario Oficial de la Asamblea Nacional. Managua, Nicaragua.

Noguera-Talavera, A. 2010. Explorando la biodiversidad: Un estudio de los ecosistemas desde la perspectiva de uso local en comunidades de cuatro áreas protegidas de Nicaragua. Informe de Investigación. Facultad de Recursos Naturales y del Ambiente, Universidad Nacional Agraria. Managua, NI. 105 p.

Pezoa, A. 2001. Estrategias de conservación de la diversidad biológica. Libro rojo de la flora nativa y de los sitios prioritarios para su conservación. Ed. Universidad de la Serena. Serena, CH. p 273-280.

Presidencia de la Republica de Nicaragua. 1998. Ley No. 290, Ley de Organización, Competencia y Procedimientos del Poder Ejecutivo. La Gaceta No. 91 y 92 Diario Oficial de la Asamblea Nacional. Managua, Nicaragua.

Rueda, R. 2007. Recopilación de la Información sobre biodiversidad en Nicaragua. Universidad Nacional Autónoma de León, NI. $204 \mathrm{p}$. 